\title{
Perioperative Fluid Management
}

\author{
Zubin M. Bamboat, M.D. ${ }^{1}$ and Liliana Bordeianou, M.D. ${ }^{1}$
}

\section{ABSTRACT}

Many colorectal surgeons rely on traditional theories and approaches in addressing perioperative fluid management issues. Often, their training and instincts favor overresuscitation, especially after bowel or emergent colorectal procedures. However, data are now emerging to support the use of more restrictive approaches to perioperative fluid administration - though uncertainties still exist as to which fluids are optimal, and how and when they should be administered. This article provides a focused, evidence-based review on this topic-highlighting critical considerations that clinicians may wish to address to improve patient outcomes following colorectal surgery.

KEYWORDS: Perioperative management, fluid restriction, fluid replacement, crystalloid, colloid

Objectives: After completing this article, the reader should be able to identify vital components of optimal fluid management in patients before, during, and following colorectal surgery.

Colorectal surgery patients, especially those who undergo extensive bowel resections and emergency laparotomies, may undergo acute alterations in volume status and in the composition of intracellular and extracellular fluids. As a result, precise perioperative management of fluids and electrolytes is a fundamental part of a patient's overall surgical treatment, which can have a significant effect on perioperative morbidity and mortality.

The fundamental goal of perioperative fluid management is on one hand-to achieve a balance between avoiding hypotension, impaired tissue oxygenation, and inadequate organ profusion that may be associated with too little fluid, and-on the other hand-to avoid interstitial edema and cardiopulmonary complications associated with fluid overload (Fig. 1). Although there is little dispute about these basic goals of perioperative fluid management, the means by which these goals are attained have been the subject of continuing debate.
The debate begins with the quantity of fluids that should be administered before, during, and after surgery. Traditionally, this quantity of fluids has been left to, and depended on, the surgeon's clinical judgment. For many decades, beginning with the onset of the modern surgical era in the early 1900s, this judgment was expressed in a marked proclivity toward an excess of intravenous fluid administration. As late as the 1960s, work by Shires popularized the notion of a "third space" fluid deficit, described as a functional loss of fluid that could not be accounted for by measurable fluid losses during bowel surgery, which must be replenished by estimated rates of intravenous fluid administration. ${ }^{1}$ Many physicians were led to infuse large quantities of fluids, going beyond measurable fluid losses, to take account of this "third space."

In the mid-1960s, commentaries on the adverse effects of indiscriminate use of saline-based solutions began to emerge. ${ }^{2}$ These commentaries were reinforced

\footnotetext{
${ }^{1}$ Harvard Medical School, Department of Surgery, Massachusetts General Hospital, Boston, Massachusetts.

Address for correspondence and reprint requests: Liliana Bordeianou, M.D., Department of Surgery, Massachusetts General Hospital, 15 Parkman St., WAC 460, Boston, MA 02114 (e-mail: lbordeianou@partners.org).
}

Perioperative Management and Anesthesia; Guest Editor, W. Brian Sweeney, M.D.

Clin Colon Rectal Surg 2009;22:28-33. Copyright (C) 2009 by Thieme Medical Publishers, Inc., 333 Seventh Avenue, New York, NY 10001, USA. Tel: +1(212) 584-4662.

DOI 10.1055/s-0029-1202883. ISSN 1531-0043. 

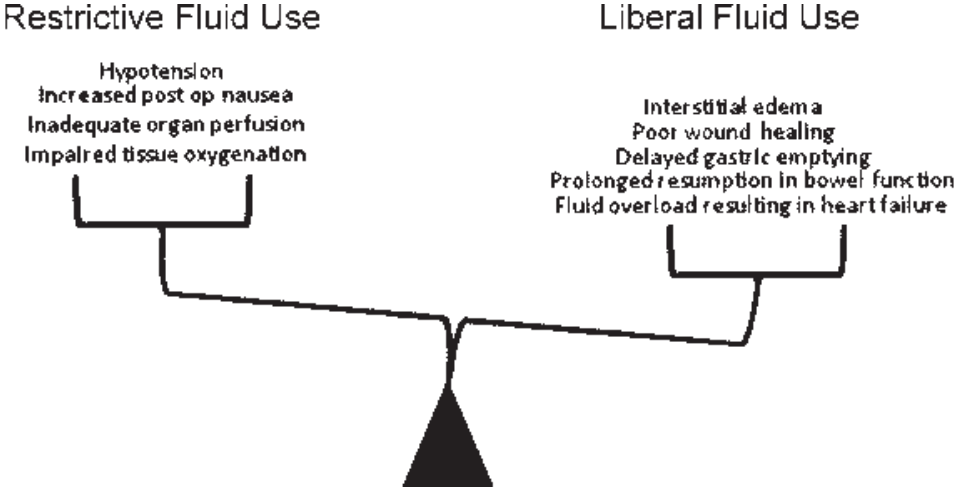

Figure 1 Adverse consequences of a restrictive or liberal approach to perioperative fluid management in patients undergoing colorectal surgery.

by studies describing complications of high volume states, such as acute respiratory distress syndrome (ARDS). ${ }^{3}$ As a result of this body of literature, the traditional approach to perioperative fluid management has been refined to take into account preoperative fluid deficits, intraoperative fluid shifts and losses, and the effect of fluid balance on the range of possible postoperative outcomes. That refinement continues as new data emerges.

\section{PREOPERATIVE CONSIDERATIONS}

The care of a surgical patient and fluid management usually begins long before an incision is made. During this preoperative stage, a surgeon seeks to determine whether a patient has a fluid deficit that may require correction prior to surgery. The assessment generally begins with a history, in which the surgeon inquires as to how long the patient has been without normal oral fluid intake and whether or not he has been exposed to extenuating circumstances or other sources of fluid loss such as vomiting. The time course and severity of any of the above factors coupled with signs of an existing fluid deficit on physical exam (dry mucous membranes, orthostasis, decreased skin turgor, etc.) provides the basis for a generalized estimate of the net preoperative fluid deficit.

One particularly significant consideration in evaluating a patient's fluid loss prior to surgery, is the presence of bowel preparation (prep). Several studies have shown that some bowel preps may cause preoperative fluid loss in patients undergoing major colorectal surgery. These studies suggest that hypertonic bowel preps (e.g., sodium phosphate) in healthy volunteers may result in dehydration, increases in plasma osmolality, urea and phosphate concentrations, and other significant changes. ${ }^{4}$ An additional consideration is the lack of oral intake after midnight experienced by most colorectal patients. Based on these alterations, some authors suggest that low levels of crystalloid replace- ment $(<500 \mathrm{~mL})$ may improve subjective sensations such as thirst, whereas large volumes of replacement (2 L) improve postoperative symptoms such as dizziness and nausea. ${ }^{5}$

With accumulating evidence in several metaanalyses supporting the notion that mechanical bowel preps (MBPs) do not improve morbidity or mortalityand in fact may increase the rates of anastomotic leakage - the use of MBPs in elective colorectal surgery is becoming less common. ${ }^{6-8}$ Eventually, this evidence may lead to a general change in practice, which, in turn, may simplify preoperative fluid management considerations. Currently, many patients still undergo bowel prep and it is prudent to assume that some of these patients are depleted of at least $500 \mathrm{~mL}$ of fluid upon entering the operating room.

Any description of preoperative fluid management considerations must also include an important cautionary note. Although measurable signs of fluid deficit may be elicited from a patient history, or from a basic physical exam, inaccuracies may result from this evaluation as the result of a process that relies largely on patient recall and subjective physician analyses. In fact, some studies evaluating experienced clinicians' estimations of degree of hypovolemia by physical exam, have suggested that an accurate diagnosis is made only $50 \%$ of the time. ${ }^{9}$ Therefore, in many cases, it will be desirable to use additional laboratory values (hemoglobin level; blood urea nitrogen [BUN]; creatinine) to corroborate clinical suspicion of fluid deficit. This is particularly true for major colorectal operations or emergent cases. Although variations in estimates on fluid balance are unlikely to have any significant impact on outcome following elective same-day surgery, major colorectal operations or emergent cases often involve greater blood loss, infectious complications, and longer operative times-with greater fluid shifts-making the optimal approach to fluid management more complex and critical to patient outcome. 


\section{INTRAOPERATIVE ASSESSMENT AND MANAGEMENT}

\section{The Traditional Approach}

Once the patient enters the operating room, the intraoperative stage of fluid management begins. Generally speaking, this stage is based on standardized formulas, which purport to account for preoperative fluid losses, intraoperative blood loss, and insensible losses. For example, many textbooks recommend that patients undergoing major abdominal surgery receive preoperative crystalloid loading at $2 \mathrm{~mL} / \mathrm{kg} / \mathrm{h}$ of fasting, and then receive infusions of crystalloids at three to four times the actual blood loss. In addition, patients are frequently administered 4 to $8 \mathrm{~mL} / \mathrm{kg} / \mathrm{h}$ of crystalloid based on suspected insensible losses, such as third spacing and evaporation. This formula may result in basal crystalloid infusion rates up to $20 \mathrm{~mL} / \mathrm{kg} / \mathrm{h}$. The rates are frequently titrated to obtain a urine output of 0.5 to $1 \mathrm{~mL} / \mathrm{kg} / \mathrm{h}^{10}$

Rates of infusion at surgery are further influenced by the effects of general anesthesia. Most general anesthetics are known to depress myocardial function. This issue is often addressed around the time of induction by the administration of additional boluses of fluid to increase stroke volume. Some inhalational agents such as isoflurane have been shown in animal models to alter the distribution of intravenous fluids favoring the interstitial compartment. ${ }^{11}$ Finally, the effects of regional anesthesia (epidural and spinal blockade) on depressing autonomic nervous function need to be considered. Although neither of these modalities on their own alters circulating blood volumes, decreases in blood pressure following their initiation are often treated by fluid administration. ${ }^{12}$ Fluid boluses to compensate for the cardiovascular effects of regional or general anesthesia are usually relatively small; however, they can play a role in the critically ill surgical patient with poor cardiopulmonary reserve.

\section{Restrictive Versus Liberal Fluid Strategies}

In general, as noted above, intraoperative fluid management formulas tend to favor a rather liberal approach to fluid replacement. However, that approach is becoming the subject of significant debate in surgical patients in general and particularly in colorectal surgery patients. To date, seven randomized trials have assessed the role of perioperative fluid restriction on a range of physiologic and functional outcomes in patients undergoing colorectal surgery. The studies have reached conflicting conclusions (Table 1), with most suggesting that the traditional, liberal approaches have adverse outcomes, and that a more restrictive approach to fluid management may be beneficial in some or all cases.

For example, one randomized, observer-blinded multicenter trial of 172 patients found that a more restricted approach to intraoperative fluid management (median $2.7 \mathrm{~L}$ on the day of operation) resulted in significantly lower rate of postoperative complications compared with a standard, more liberal regimen $(5.4 \mathrm{~L})$ that resembled the prevailing practice. ${ }^{13}$ This was manifested by decreased cardiopulmonary and wound healing adverse events and a trend toward fewer deaths in the restricted fluid treatment arm.

Likewise, a second prospective study-looking at the impact of two intraoperative fluid regimens on the outcome of 152 patients undergoing intraabdominal surgery - found that patients managed with a restrictive

Table 1 Summary of Existing Trials Investigating the Effect of Fluid Restriction during the Perioperative Period in Patients Undergoing Colorectal Surgery

\begin{tabular}{|c|c|c|c|c|c|}
\hline Author & Study Design & $\begin{array}{l}\text { Perioperative } \\
\text { Period Studied }\end{array}$ & $\begin{array}{l}\text { Volume } \\
\text { Administered }\end{array}$ & $\begin{array}{l}\text { Effect of Fluid } \\
\text { Restriction }\end{array}$ & Primary Endpoints/Results \\
\hline Brandstrup $^{13}$ & $\begin{array}{l}\text { DB, Randomized } \\
\text { MCT }\end{array}$ & $\begin{array}{l}\text { Intraoperative } \\
\text { and postoperative }\end{array}$ & 2.7 L vs. $5.4 \mathrm{~L}$ & Beneficial & $\begin{array}{l}\text { Fewer complications in } \\
\text { restricted group }\end{array}$ \\
\hline Nisanevich $^{14}$ & $\mathrm{RCT}$ & Intraoperative & 1.2 L vs. $3.7 \mathrm{~L}$ & Beneficial & $\begin{array}{l}\text { Mortality no different } \\
\text { Fewer complicatins in restrictive group }\end{array}$ \\
\hline Holte $^{15}$ & $\mathrm{DB}, \mathrm{RCT}$ & Intraoperative & $1.6 \mathrm{~L}$ vs. $5 \mathrm{~L}$ & Beneficial & $\begin{array}{l}\text { Pulmonary funciion improved } \\
\text { in restrictive group }\end{array}$ \\
\hline Mackay $^{25}$ & $\mathrm{DB}, \mathrm{RCT}$ & Postoperative & 4.5 L vs. 8.75 L & No Effect & Hospital length of stay unchanged \\
\hline Campbell $^{10}$ & $\mathrm{RCT}$ & Intraoperative & $\begin{array}{r}5-10 \mathrm{~mL} / \mathrm{kg} / \mathrm{h} \text { vs. } \\
10-15 \mathrm{~mL} / \mathrm{kg} / \mathrm{h}\end{array}$ & Harmful & $\begin{array}{l}\text { Reduced hemodynamic } \\
\text { stability and urine output in } \\
\text { restrictive group }\end{array}$ \\
\hline Arkilic ${ }^{27}$ & $\mathrm{RCT}$ & Intraoperative & $2.8 \mathrm{~L}$ vs. $5 \mathrm{~L}$ & Harmful & $\begin{array}{l}\text { Decreased tissue perfusion } \\
\text { and oxygen tension in } \\
\text { restrictive group }\end{array}$ \\
\hline
\end{tabular}

$\mathrm{DB}$, double blind; $\mathrm{MCT}$, multicenter trial; $\mathrm{RCT}$, randomized controlled trial. 
fluid approach (median 1.2 L versus 3.7 L) experienced fewer complications (17 versus $31 \%$ ), earlier resumption of bowel function, and shorter lengths of stay. ${ }^{14}$

Additional observations of the deleterious effect of high fluid repletion rates on colorectal surgery patients have been noted in patients who are on fast track postoperative recovery protocols. For example, a recent double blind study comparing the effects of a restrictive $(\sim 1.6 \mathrm{~L})$ versus a liberal $(\sim 5 \mathrm{~L})$ intraoperative fluid strategy in patients undergoing fast track recoveries after colon surgery revealed that the restrictive group had improved postoperative pulmonary function and hypoxemia with a trend toward fewer complications. ${ }^{15}$

On the other hand, at least one randomized study found that liberal intravenous (IV) fluid administration during surgery may have an important functional benefit. This study assigned patients undergoing bowel resection to either a liberal (10 to $15 \mathrm{~mL} / \mathrm{kg} / \mathrm{h}$ ) or restrictive intraoperative fluid regimen $(5$ to $10 \mathrm{~mL} / \mathrm{kg} / \mathrm{h}){ }^{10}$ The authors then reported that the patients treated restrictively manifested increased hemodynamic instability as measured by changes in arterial blood pressure and intraoperative urine output. They concluded that more liberal approaches to intraoperative fluid management may have a beneficial effect on cardiopulmonary and renal function in patients undergoing bowel resection, and that the restrictive approach to fluid management might have unintended consequences for patients.

All of these data need to be interpreted with caution. The studies did not use consistent definitions of "restrictive" versus "liberal" IV fluid management, and, as a result, the guidelines that emerge from these studies-as to what would constitute safe restrictive management-are far less well defined than those expressed in the more traditional protocols. These conflicting studies suggest that the issue of whether or not to use liberal or restrictive approaches to intraoperative fluid management is far from resolved at this time. What can be concluded thus far is that fluid management strategies cannot be based on inflexible formulas and should instead, be individualized. Factors to consider include the type of surgery, length of the operation, patient's preexisting comorbidities, and their predicted postoperative course.

\section{Guiding Fluid Therapy}

Measurements of the effective circulating blood volume during surgery can be made using parameters such as blood loss, urine output, third space, and insensible losses; nevertheless, they serve only as a rough estimate. In most cases of colorectal surgery, these parameters are adequate indices with which the clinician can make informed decisions on intraoperative fluid management. Important caveats to keep in mind when using urine output as a marker of adequate blood volume and tissue perfusion include:
1. Low urine output does not always correlate with low blood volume, as the natural stress response to surgery is an increase in sympathetic tone and the release of antidiuretic hormone, both of which can lower urine output. ${ }^{16}$

2. The heart and kidneys that serve to recognize changes in circulating blood volume do so via signaling mediated by stretch receptors. Activation of these receptors in response to changes in blood flow alone is possible and therefore may not always result from alterations in blood volume.

3. There is very scant data in the literature that supports the notion that low intraoperative urine output is a marker of subsequent renal dysfunction. ${ }^{17}$

Other more invasive means of determining blood volume and guiding fluid management include the use of pulmonary artery (PA) catheters and central venous pressure (CVP) lines. Although both of these methods continue to be used in critically ill patients during and after major abdominal operations, they are fraught with confounding effects. Both modalities are affected by patient position, ventricular function, pulmonary compliance and ventilator settings, and drugs that alter vascular tone. It is therefore not surprising that despite being armed with both these tools, critical care physicians are still not able to accurately determine blood volume. ${ }^{18}$ Whether or not alterations in fluid management based on central hemodynamic monitoring affects outcome following surgery was an issue of controversy. Now the general consensus based on a series of large randomized controlled trials (RCTs) and a subsequent meta-analysis is that while more fluid-based interventions occur in response to changes in central hemodynamic parameters, survival is unaffected. ${ }^{19}$

\section{Choice of Fluids}

A final, important detail pertaining to intraoperative fluid management is the choice of fluids to be administered as replacement for the intraoperative losses incurred. The primary goal of fluid administration is to restore the effective circulating blood volume to a level that ensures adequate tissue oxygenation. This restoration must occur rapidly, while minimizing potential adverse consequences such as fluid accumulation in the interstitial space. The administration of crystalloids, colloid and blood products must attain a desirable equilibrium between the various fluid compartments within the body. Various neurohormonal pathways within the body become activated following the induction of surgical stress and fluid shifts.

Initially, administration of salt-containing solutions during surgery was thought to be detrimental as the body was thought to become "salt intolerant" in the postoperative period secondary to activation of the 
renin-angiotension pathway. ${ }^{20}$ For a while, sodium free replacements were advocated. However, the dangerous effects of decreasing plasma sodium levels via the administration of dextrose-based, sodium-free solutions later became evident. In addition, the ability of sodiumcontaining regimens to maintain intravascular oncotic pressure led to the current practice of administering isoosmotic crystalloid solutions during the perioperative period. $^{21}$

The second, more recent debate, arose around the issue of whether colloid infusions (albumin, hydroxyethyl starch, modified gelatin, or dextran) may be more efficient in restoring intravascular volume than isotonic crystalloid infusions. However, the most recent Cochrane Database Review addressing the effect of crystalloids versus colloids in the treatment of critically ill trauma, burn, and surgical patients revealed that there is no evidence that administration of colloids reduces the risk of death, pulmonary edema, or length of stay. ${ }^{22}$ Given their significantly higher cost, colloids are used on an individual specific basis and increasingly sparingly in routine colorectal surgery. Currently there are no data to support the use of any particular colloid over another when it comes to impact on survival. ${ }^{23}$

\section{POSTOPERATIVE CONSIDERATIONS}

There seems to be least debate - at least for now - as to liberal versus restrictive fluid management in the post- operative period. To date, these data seem to clearly point in favor of a more restrictive approach. In a randomized prospective study in which patients were assigned to either a restricted $(<2 \mathrm{~L})$ or standard $(>3 \mathrm{~L})$ postoperative intravenous fluid regimen, patients receiving less IV fluids were found to have earlier gastric emptying, a shorter time to passage of flatus and first bowel movement. ${ }^{24}$ Conversely, the group of patients treated with the standard liberal formula of IV fluid repletion had longer hospital stays and more complications. ${ }^{24}$

A similar study by MacKay et al investigated the effects of a liberal (median $8.75 \mathrm{~L}$ ) versus a restricted (median $4.5 \mathrm{~L}$ ) postoperative fluid regimen in 80 randomized patients following elective colorectal surgery. In contrast to the study above, they found no differences between the treatment groups with respect to median time to passage of flatus, resumption of bowel function, or length of stay, but also no harm in a more restrictive resuscitation approach. ${ }^{25}$

A restrictive approach to fluid management in the postoperative period makes sense. Weight gain and edema as a result of fluid administration have been associated with adverse outcomes following colorectal surgery. ${ }^{13,14}$ Tissue edema in particular has been shown to correlate with adverse outcomes such as poor wound healing, compromised pulmonary function and delayed resumption of bowel function. ${ }^{13,26}$ Furthermore, many patients are already mobilizing the excess fluids they may have received intraoperatively. In this setting, IV fluid

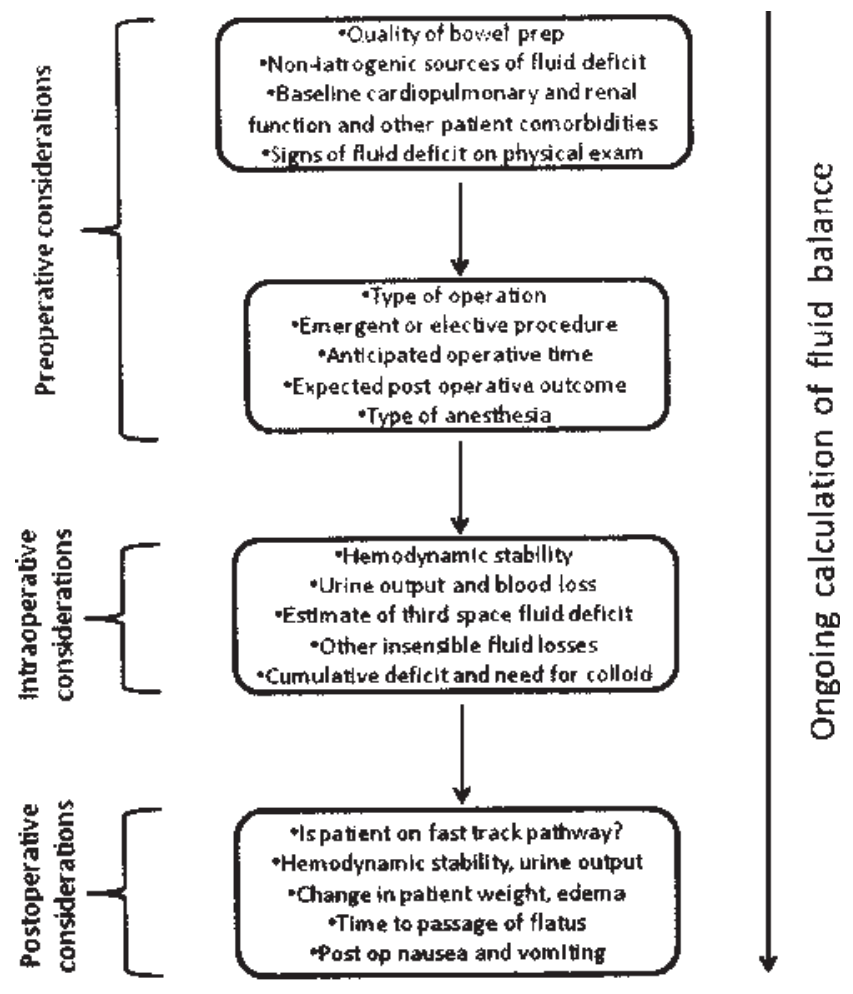

Figure 2 Perioperative fluid management decision pathway. Consideration of these factors will enable clinicians to make an accurate ongoing assessment of the net fluid deficit/excess in colorectal surgery patients. 
repletion based on an hour to hour urine output measurement without consideration of the patient's overall volume status as manifested by such measurements as body weight, blood pressure, heart rate, drain outputs and hemoglobin measurements is not justified.

\section{SUMMARY}

Traditional methods of perioperative fluid management have emphasized a liberal approach to administration of fluids. Those traditional methods have now been called into question. So, should the standard of care for patients undergoing colorectal surgery utilize a restrictive rather than a liberal perioperative fluid regimen?

The answer is unclear. Additional trials with larger patient cohorts, focusing on functional outcomes as primary endpoints are needed. However, the prevailing trend-supported by data from several RCTs-is to favor the restrictive approach that appears to have a positive impact on patient outcome. That said, a standard "cookbook" strategy to fluid management may be inadequate for some surgical patients. For the moment, the best approach may be an individualized form of treatment, based on a variety of patient-specific clinical considerations (Fig. 2).

\section{REFERENCES}

1. Shires T, Williams J, Brown F. Acute change in extracellular fluids associated with major surgical procedures. Ann Surg 1961;154:803-810

2. Shields CJ. Towards a new standard of perioperative fluid management. Ther Clin Risk Manag 2008;4(2):569571

3. Petty TL, Ashbaugh DG. The adult respiratory distress syndrome. Clinical features, factors influencing prognosis and principles of management. Chest 1971;60(3):233-239

4. Holte K, Nielsen KG, Madsen JL, Kehlet H. Physiologic effects of bowel preparation. Dis Colon Rectum 2004;47(8): 1397-1402

5. Holte K, Kehlet H. Compensatory fluid administration for preoperative dehydration-does it improve outcome? Acta Anaesthesiol Scand 2002;46(9):1089-1093

6. Bucher P, Mermillod B, Gervaz P, Morel P. Mechanical bowel preparation for elective colorectal surgery: a meta-analysis. Arch Surg 2004;139(12):1359-1364; discussion 1365

7. Nichols RL, Choe EU, Weldon CB. Mechanical and antibacterial bowel preparation in colon and rectal surgery. Chemotherapy 2005;51(Suppl 1):115-121

8. Wille-Jorgensen P, Guenaga KF, Matos D, Castro AA. Preoperative mechanical bowel cleansing or not? an updated meta-analysis. Colorectal Dis 2005;7(4):304-310

9. Chung HM, Kluge R, Schrier RW, Anderson RJ. Clinical assessment of extracellular fluid volume in hyponatremia. Am J Med 1987;83(5):905-908
10. Campbell IT, Baxter JN, Tweedie IE, Taylor GT, Keens SJ. IV fluids during surgery. Br J Anaesth 1990;65(5):726-729

11. Connolly CM, Kramer GC, Hahn RG, et al. Isoflurane but not mechanical ventilation promotes extravascular fluid accumulation during crystalloid volume loading. Anesthesiology 2003;98(3):670-681

12. Lobo DN, Stanga Z, Simpson JA, et al. Dilution and redistribution effects of rapid 2-litre infusions of $0.9 \%(\mathrm{w} / \mathrm{v})$ saline and $5 \%(\mathrm{w} / \mathrm{v})$ dextrose on haematological parameters and serum biochemistry in normal subjects: a double-blind crossover study. Clin Sci (Lond) 2001;101(2):173-179

13. Brandstrup B, Tonnesen H, Beier-Holgersen R, et al. Effects of intravenous fluid restriction on postoperative complications: comparison of two perioperative fluid regimens: a randomized assessor-blinded multicenter trial. Ann Surg 2003;238(5):641-648

14. Nisanevich V, Felsenstein I, Almogy G, et al. Effect of intraoperative fluid management on outcome after intraabdominal surgery. Anesthesiology 2005;103(1):25-32

15. Holte K, Foss NB, Andersen J, et al. Liberal or restrictive fluid administration in fast-track colonic surgery: a randomized, double-blind study. Br J Anaesth 2007;99(4):500-508

16. Fieldman NR, Forsling ML, Le Quesne LP. The effect of vasopressin on solute and water excretion during and after surgical operations. Ann Surg 1985;201(3):383-390

17. Alpert RA, Roizen MF, Hamilton WK, et al. Intraoperative urinary output does not predict postoperative renal function in patients undergoing abdominal aortic revascularization. Surgery 1984;95(6):707-711

18. Barker SJ. Blood volume measurement: the next intraoperative monitor? Anesthesiology 1998;89(6):1310-1312

19. Shah MR, Hasselblad V, Stevenson LW, et al. Impact of the pulmonary artery catheter in critically ill patients: metaanalysis of randomized clinical trials. JAMA 2005;294(13): 1664-1670

20. Coller FA, Campbell KN, Vaughan HH, Iob LV, Moyer CA. Postoperative Salt Intolerance. Ann Surg 1944;119(4): 533-541

21. Roberts JP, Roberts JD, Skinner C, et al. Extracellular fluid deficit following operation and its correction with Ringer's lactate. A reassessment. Ann Surg 1985;202(1):1-8

22. Perel P, Roberts I. Colloids versus crystalloids for fluid resuscitation in critically ill patients. Cochrane Database Syst Rev 2007;(4):CD000567

23. Bunn F, Trivedi D, Ashraf S. Colloid solutions for fluid resuscitation. Cochrane Database Syst Rev 2008;(1): CD001319

24. Lobo DN, Bostock KA, Neal KR, et al. Effect of salt and water balance on recovery of gastrointestinal function after elective colonic resection: a randomised controlled trial. Lancet 2002;359(9320):1812-1818

25. MacKay G, Fearon K, McConnachie A, et al. Randomized clinical trial of the effect of postoperative intravenous fluid restriction on recovery after elective colorectal surgery. Br J Surg 2006;93(12):1469-1474

26. Grocott MP, Mythen MG, Gan TJ. Perioperative fluid management and clinical outcomes in adults. Anesth Analg 2005;100(4):1093-1106

27. Arkilic CF, Taguchi A, Sharma N, et al. Supplemental perioperative fluid administration increases tissue oxygen pressure. Surgery 2003;133:49-55 